\title{
Contaminación del agua por metales pesados, métodos de análisis y tecnologías de remoción. Una revisión ${ }^{1}$
}

\section{Water contamination by heavy metals, analysis methods and removal technologies. A review}

\author{
S. E. Pabón, R. Benítez, R. A. Sarria-Villa y J. A. Gallo
}

Recibido: noviembre 21 de 2018 - Aceptado: enero 15 de 2020

\begin{abstract}
Resumen- Los sistemas de producción industrial actuales usan metales pesados para la extracción de un material o como elemento en la refinación de un producto en particular lo que en principio resulta en la obtención del producto deseado con relativos bajos costos de producción. Sin embargo, usar estos metales presenta un grave problema a nivel ambiental debido a sus altos niveles de toxicidad para los organismos con los que interactúen una vez son descargados. Por tanto, se han desarrollado diversos métodos para tratar con estos metales una vez están en el agua y los métodos para analizarlos. Se realizó una búsqueda amplia y actualizada de las fuentes, efectos y toxicidad en los seres vivos, métodos de análisis y las diversas técnicas de tratamiento existentes para metales pesados. Como resultado el investigador estará más preparado al tener información actual sobre los tratamientos a tener en cuenta en la manipulación de metales pesados.

Palabras clave - contaminación, fuentes hídricas, mercurio, métodos de análisis, técnicas de remoción.
\end{abstract}

1 Producto derivado del proyecto de investigación "Optimización de la síntesis de nanopartículas de óxido de hierro usando extracto de Eucalyptus grandis y evaluación de su posible actividad en la retención de Hg en agua". Desarrollado en los laboratorios del Grupo de Investigación en Química Analítica Ambiental y la Unidad de Análisis Industriales del Departamento de Química de la Universidad del Cauca.

S. E. Pabón, Universidad del Cauca, Popayán, Colombia, email: santiagopabon@unicauca.edu.co.

R. Benitez, Universidad del Cauca, Popayán, Colombia, email: rbenitez@unicauca.edu.co.

R. A. Sarria-Villa, Universidad del Cauca, Popayán, Colombia, email: rodrigosv@unicauca.edu.co.

J. A. Gallo, Universidad del Cauca, Popayán, Colombia, email: jagallo@unicauca.edu.co.

Como citar este artículo: Pabón, S. E., Benitez, R., Sarria-Villa R. A., Gallo, J. A. Contaminación del agua por metales pesados, métodos de análisis y tecnologías de remoción. Una revisión, Entre Ciencia e Ingeniería, vol. 14, no. 27, pp. 9-18, enero-junio 2020. DOI: https://doi.org/10.31908/19098367.1734.

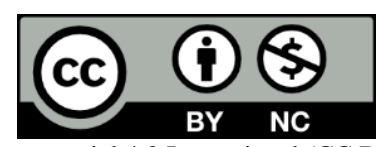

Attribution-NonCommercial 4.0 Intenational (CC By-NC 4.0)
Abstract - Current industrial production systems nowadays use heavy metals for the extraction of a material or as an element in the refining of a particular product which in principle results in obtaining the desired product with relatively low production cost. However, using these metals presents a serious environmental problem due to their high levels of toxicity for the organisms which they interact once they are discharged. Therefore, various methods have been developed to deal with these metals once they are in the water and the methods to analyze them. A comprehensive and update search was made of the sources, effects and toxicity in the living beings, analysis methods and the various techniques of heavy metal treatment. As a result, the researcher will be more prepared to have current information on the treatments to be taken into account in the handling of heavy metals.

Keywords - contamination, water sources, mercury, analysis methods, removal techniques.

\section{INTRODUCCIÓN}

$\mathrm{L}$ os metales pesados son considerados según la tabla periódica como elementos químicos de alta densidad (mayor a $4 \mathrm{~g} / \mathrm{cm}^{3}$ ), masa y peso atómico por encima de $20 \mathrm{y}$ son tóxicos en concentraciones bajas. Algunos de estos elementos son Aluminio ( $\mathrm{Al})$, berilio $(\mathrm{Be})$, Cobre $(\mathrm{Cu})$, Hierro $(\mathrm{Fe})$, Manganeso (Mn), Cadmio (Cd), Mercurio ( $\mathrm{Hg})$, plomo $(\mathrm{Pb})$, entre otros [1]. Actualmente uno de los mayores problemas a nivel ambiental es la contaminación de las fuentes hídricas del mundo por metales pesados, ya que es debido a la toxicidad que presentan los metales pesados en el agua de los ríos, que son considerados como un serio problema para los habitantes de las poblaciones que se abastecen de dichos ríos en especial si se considera que el incremento en la concentración de estos metales en las fuentes hídricas procede de las diversas actividades antropogénicas, elevando además los efectos potencialmente nocivos sobre los diferentes sistemas ecológicos y el ambiente, los cuales son el soporte de la vida humana lo cual acarrea serios problemas a nivel económico tanto a nivel local como nacional debido al aumento en los costos de los tratamientos médicos y una disminución en la productividad de los habitantes de la zona [2], [3]. 
Dentro de los principales factores que afectan los ecosistemas por contaminación por metales pesados, se encuentra las actividades humanas donde destacan como mayoritarias las operaciones de tipo minero y de fundición entre otras actividades de tipo industrial y urbana [4], donde se tiene que la tasa de contaminación del agua puede rondar cerca de los 200 millones de metros cúbicos diarios [5]. Este hecho conlleva un gran número de problemas tanto en la vida de las plantas ya que estos metales acaban depositados en los suelos transportados hasta los mismos por los ríos, como por ejemplo la disminución del crecimiento o el amarillamiento de las hojas (clorosis) [6], como en la vida humana donde los efectos pueden ser erupciones cutáneas, malestar de estómago y ulceras, problemas respiratorios, debilitamiento del sistema inmune, daño en los riñones e hígado, cáncer de pulmón, afecciones cardiacas, óseas, testiculares y del sistema nervioso central y periférico o la muerte [7], [8].

Debido a esta problemática para los investigadores se ha tornado de suma importancia encontrar diversos métodos para la retención y extracción de dichos metales de las fuentes hídricas y reducir la toxicidad en las mismas para garantizar la preservación tanto de los ecosistemas y la vida humana. Entre los diversos métodos existentes para el control de este tipo de metales podemos encontrar métodos tales como: precipitación, oxido-reducción, intercambio iónico, filtración, tratamiento electroquímico, tecnologías de membrana y recuperación por evaporación, adsorción y bioadsorción [9]. Dentro de los metales que se consideran como pesados están el plomo, el estaño, el hierro, el cadmio, el mercurio, cromo, vanadio, entre otros [10], [11]. En este artículo se describe algunas de las fuentes de metales pesados, sus efectos a nivel de un ecosistema y la salud humana y los diferentes métodos existentes para su tratamiento.

\section{FUENTES DE METALES PESADOS}

Los metales pesados como plomo, cadmio, cromo, zinc, mercurio entre otros, son liberados hacia ecosistemas acuáticos así como a los suelos principalmente debido a diversas actividades antropogénicas y presenta una seria amenaza para las plantas, animales e incluso los humanos debido a su persistencia, bioacumulación, propiedad no biodegradable y su toxicidad incluso a bajas concentraciones [12], [13]. El hecho de que estos metales se encuentren en los diversos ecosistemas es de preocuparse, dado que muchos seres vivos dependen del adecuado equilibrio en su lugar de alimentación o de vivienda, el cómo llegan dichos metales a los distintos ecosistemas varía según el tipo de actividad que se desarrolla por el hombre, por ejemplo el cromo que es un compuesto ampliamente usado en la industria en áreas como el revestimiento plástico, galvanoplastia de metales para resistencia de la corrosión, curtido y acabado de cueros, en pigmentos y para conservantes de madera [14], otro tipo de metales como el cadmio son usados en la industria para la fabricación de baterías de níquel-cadmio, agentes anticorrosivos y pigmentos [15]. Para el mercurio se han identificado seis fuentes de contaminación que son la deposición atmosférica, erosión, descargas humanas, materiales agrícolas, minería y las descargas industriales y de combustión, como tal en las aguas subterráneas naturales y aguas superficiales el contenido de este metal está por debajo de $0.5 \mu \mathrm{g} / \mathrm{L}$, sin embargo en aguas residuales descargadas cerca de depósitos minerales locales y otros sitios contaminados la concentración de mercurio es mucho mayor [16]. El plomo también es liberado al ambiente debido a la industria minera y la quema de combustibles fósiles, está involucrado en la fabricación de baterías, municiones, productos metálicos y dispositivos para la protección contra los rayos X [17].

Como se puede ver el mayor aporte a la contaminación de las aguas por metales pesados es debido a las descargas de aguas residuales producto de diversas actividades antropogénicas principalmente de tipo industrial, cada día es mayor el grado de contaminación de las fuentes hídricas debido al constante uso de estos metales en la industria, lo que aumenta la concentración de los mismos en el agua y por ende aumenta el riesgo para los seres humanos y demás organismos vivos al aumentar la probabilidad de quedar expuestos a estos metales, lo cual es de preocupar si consideramos que los niveles permisibles en el agua para consumo humano de metales como el mercurio, plomo y cromo son de $1.0 \mu \mathrm{g} / \mathrm{L}, 10$ $\mu \mathrm{g} / \mathrm{L}$ y $50 \mu \mathrm{g} / \mathrm{L}$ respectivamente según la legislación $\mathrm{RD}$ 140/03 y la NTC 183 [18], [19], mientras que se establece un valor máximo de $2.0 \mu \mathrm{g} / \mathrm{L}, \quad 15 \mu \mathrm{g} / \mathrm{L} \quad$ y $100 \quad \mu \mathrm{g} / \mathrm{L}$ respectivamente según la agencia de protección ambiental de estados unidos (EPA) [20].

\section{EFECTOS SOBRE LOS SERES VIVOS Y TOXICIDAD}

Es evidente el hecho de que la principal fuente de metales pesados radica en las actividades industriales realizadas por la humanidad, esto afecta de forma considerable no solo la vida humana si no a los animales, las plantas, las fuentes hídricas y los suelos, en otras palabras, afecta toda la cadena trófica debido a la descarga de metales pesados al ambiente. De hecho, en diferentes partes de Colombia es frecuente encontrar reportes de metales pesados asociados a la industria, la actividad agrícola y la minería de la siguiente manera: mercurio en fuentes de agua (quebradas, lagunas) de Meta, Boyacá y Antioquia. Arsénico en cultivos como hortalizas y legumbres en Nariño, diversos vegetales y aguas contaminados con plomo y mercurio en Chocó, así como uso indiscriminado de mercurio y cianuro en explotación minera, especialmente de oro en el Nordeste y Bajo Cauca (Antioqueño), Marmato (Caldas) y en más de 500 minas de todo el país [1]. Como tal, una vez el metal pesado entra en un ecosistema inicia un proceso de cambios en todo el entorno, es decir en los suelos, en el agua y en los seres vivos que habitan en el medio, por ejemplo si tomamos el suelo como medio sobre el cual se da la contaminación por metales pesados, estos tienen diferentes rutas de acción una vez en el medio como lo serian la complejación de estos con ligandos orgánicos, con otros aniones como el $\mathrm{F}^{-}, \mathrm{SO}_{4}{ }^{2-}, \mathrm{PO}_{4}{ }^{3-}$, o que precipiten como minerales, también puede darse el caso en que 
estos metales sufren volatilización siendo liberados hacia la atmosfera, o ser adsorbidos sobre la superficie de arcillas y humus volviéndose un elemento disponible para ser asimilado por la vegetación y por tanto entrando en la cadena trófica, finalmente puede darse una transformación de los metales pesados cambiando a otro tipo de contaminante o que coprecipite junto con otros elementos, si bien hablamos del efecto que se da si la contaminación se da sobre los suelos no se debe olvidar que existe la posibilidad de que estos metales se movilicen entrando en aguas de drenaje y ríos [21], esto implica que los metales pesados serán transportados y viajaran hasta contaminar una nueva área repitiendo el proceso indefinidamente. Como se mencionó anteriormente los metales pesados tienen un serio problema en cuanto a toxicidad se refiere, del mismo modo cada institución gubernamental ha establecido los parámetros limitantes en cuanto al contenido permisible de los mismos metales en diferentes fuentes (agua, suelos, lodos, aire) además de los niveles de tolerancia que se han establecido en cuanto a la presencia de estos al interior de los organismos.

Algunos de los metales tóxicos, inducen peligrosos y tóxicos efectos sobre el ambiente en el que vivimos. A nivel humano los metales pesados como cobalto o manganeso, presentan problemas graves e irreversibles para la salud debido a su toxicidad, como por ejemplo trastornos del sistema nervioso, intoxicación crónica y aguda y pérdida de funciones orgánicas [22]. Esto hace de la exposición a estos metales un problema serio para los humanos, en especial para aquellos cuyos emplazamientos radican cerca de zonas donde el uso de dichos metales sea de forma indiscriminada y con bajos controles ambientales. Las formas iónicas de algunos de estos metales como $\mathrm{Pb}^{2+}, \mathrm{Hg}^{2+}$ reaccionan con las biopartículas al interior del cuerpo y forman compuestos tóxicos los cuales son cruciales que se aíslen [23].

En las plantas, si bien algunos metales son esenciales para su desarrollo normal pues son componentes y/o catalizadores para proteínas y enzimas $\left(\mathrm{Mn}^{2+}, \mathrm{Fe}^{3+}, \mathrm{Zn}^{2+}, \mathrm{Cu}^{2+}\right)$ y otros metales pese a no ser esenciales si resultan beneficiosos para las plantas $\left(\mathrm{Cr}^{3+}, \mathrm{V}, \mathrm{Ti}, \mathrm{Co}, \mathrm{Se}\right)$, se debe tener en cuenta que dada la alta actividad humana se liberan, en especial al suelo, grandes cantidades de metales. Este exceso de metales como Aluminio, Mercurio, Plomo, entre otros resulta de especial relevancia toxica para las plantas, en especial en suelos ácidos donde la fitotoxicidad por metales tóxicos se manifiesta particularmente tanto en el crecimiento como en la formación de raíces laterales y secundarias [24]. Además de que algunos de estos metales suponen peligros adicionales ya que como se mencionó anteriormente se integran en la cadena trófica.

El cómo medir la toxicidad provocada por los metales pesados en el caso de suelos generalmente se lleva a cabo por medio del método llamado convencional el cual consiste en la extracción de estos metales por acción de ácidos fuertes, aunque dicho método presenta su principal defecto en que solo tiene en cuenta las concentraciones totales, esto presenta la limitación de que en los suelos es posible que solo las fracciones móviles de los metales pesados sean susceptibles de ser absorbidas por las plantas lo que las hace ser consideradas como biodisponibles [25], del mismo modo entra a jugar un papel importante la movilidad de los metales pesados en el agua lo cual pasaría a ser un factor que condiciona el contenido de metales en el suelo en determinadas épocas del año si es que las plantaciones se encuentran cerca de ríos o quebradas de las cuales se alimentan.

De manera más específica se pueden realizar diversos tipos de ensayos para medir la toxicidad de los metales tanto en suelos como en aguas siendo una forma de lograrlo mediante bioensayos. En el caso de los suelos fácilmente se puede dar dicha medición por medio de la prueba de germinación de semillas/elongación de raíz, la cual evalúa el efecto fitotóxico en la germinación de las semillas y el crecimiento inicial de las plántulas, mientras que en aguas un ensayo de tipo biológico puede darse por medio de la prueba de microtox que se basa en la reducción de la luminiscencia en el tiempo luego de mezclar una bacteria acuática (Vibrio fischeri) con fases acuosas [26].

Del mismo modo existen estudios que se realizan con el fin de medir el efecto de la toxicidad de los metales pesados en organismos mayores para determinar el límite que pueden resistir, tal como la dosis letal $50\left(\mathrm{LD}_{50}\right)$, la cual hace referencia a la cantidad de un material que se da una vez que causa la muerte del $50 \%$ de la población de un grupo de animales de prueba. Generalmente esta prueba para medir la toxicidad se realiza en ratas y ratones y se expresa en la cantidad del químico suministrado (miligramos) por 100 gramos (para un animal pequeño) o por kilogramo (para sujetos de prueba más grandes) del peso corporal del animal de prueba. Puede determinarse por cualquier ruta de administración, siendo la dérmica y oral, las rutas más comunes [27].

Es entonces crucial tener presentes los valores de toxicidad de los metales pesados, en especial si los asentamientos humanos se encuentran cerca de zonas donde las actividades realizadas promueven la contaminación con metales pesados debido a los procesos que efectúan en los cuales este tipo de metales son muy usados. Para ello se recomienda tener presentes los límites permisibles de este tipo de metales en diferentes materiales como lo son el suelo, el agua, los lodos entre otros y que la comunidad internacional ya ha establecido dichos parámetros en oficinas gubernamentales como la EPA, el ministerio de ambiente entre otras.

\section{MÉTOdos DE ANÁLISIS}

Como tal los metales se encuentran fácilmente en las aguas y algunos de ellos incluso son considerados esenciales para la vida, están los que por el contrario son tóxicos y de gran impacto ambiental y son los metales pesados que como se ha mencionado antes, su particular característica es poseer una densidad considerablemente alta, casos como el titanio (Ti) el cual presenta una densidad de $4.5 \mathrm{~g} / \mathrm{cm}^{3}$ y es considerado un metal pesado o el arsénico (As) el cual si bien no es un metal, si presenta una alta densidad de $5.7 \mathrm{~g} / \mathrm{cm}^{3}$ por lo cual se le considera un metal pesado, además de que presentan otras propiedades más de carácter ambiental [28]-[30]. 
Debido a la alta contaminación que se genera se vuelve imperativo encontrar adecuados métodos de análisis que permitan un adecuado y exacto conocimiento de los niveles de un metal en el agua en especial si dichas aguas están cerca de zonas de alta actividad industrial y son fuente de suministro del recurso para comunidades aledañas con el fin de determinar la calidad del agua que se consume. Es entonces que técnicas como la absorción atómica, las técnicas fotométricas, electroquímicas entre otras surgen como alternativa para ayudar en la determinación del contenido de metales en el agua.

\section{A. Espectrometría de Absorción Atómica}

Puede ser una de las técnicas más usadas en el ámbito de la determinación del contenido de metales en una muestra. La técnica de absorción es ampliamente usada para la investigación en laboratorios, así como en la industria alimenticia, investigación ambiental, farmacéutica, y otros sectores. Como tal esta técnica presenta la particularidad de permitir la atomización de la muestra a analizar por medio de tres procesos diferentes los cuales son: espectrometría de absorción atómica por flama (FAAS), por atomización electrotérmica (ETAAS) y por generación de vapores químicos o de hidruros (CVG-AAS) [31]-[33]. Sin embargo, en la elección de la técnica adecuada entre los factores que afectan: están la naturaleza quimia del analito y su contenido en el tipo de muestra a analizar, además de la composición de la muestra y su estado físico. Si bien la técnica más usada es la técnica por llama, esta presenta poca sensibilidad respecto a las otras dos y en el caso de los metales pesados los cuales se encuentran generalmente en bajas cantidades de forma natural, la técnica de llama no es la más adecuada.

Por otro lado, está la técnica electrotérmica, en la cual se pueden analizar muestras solidas directamente y presenta mayor sensibilidad que la técnica por flama, sin embargo, requiere de una eficiente optimización de la temperatura programada, además de la elección de modificadores químicos adecuados para obtener resultados válidos.

Finalmente la última técnica es la generación de hidruros o vapores químicos la cual posee gran sensibilidad, trabaja por medio de la formación de hidruros de los metales a analizar los cuales luego son atomizados para su medición, esta técnica se ha visto restringida a cierto grupo de metales que se consideran más difíciles de trabajar en las otras técnicas de absorción atómica como $\mathrm{As}, \mathrm{Sb}, \mathrm{Se}, \mathrm{Bi}, \mathrm{Cd}, \mathrm{Pb}$ entre otros y en el caso del $\mathrm{Hg}$, se desarrolló una técnica derivada de la generación de hidruros la cual se conoce como vapor frio, la cual es una de las más recomendadas para la determinación de mercurio [34].

\section{B. Espectrometría de plasma de acoplamiento inductivo}

Como tal esta técnica espectral es un método conjunto, es decir suele ir de la mano con otros métodos como lo son la espectrometría de masas, la espectroscopia de emisión óptica, entre otros [35], [36]. Donde el plasma permite el análisis de los analitos que son ionizados en la flama conocida como el plasma sobre el cual se realiza el análisis a diferentes alturas según el analito, sin embargo es una técnica que consume en exceso el gas que forma el plasma que generalmente es argón lo cual hace que el uso de técnicas acopladas con plasma tiendan a ser costosas [37].

\section{Técnicas fotométricas.}

No siempre se cuenta con los equipos como para llevar a cabo análisis de alta eficiencia en cuanto al poder de detección de los equipos como por ejemplo con la espectrometría de absorción atómica vista en la sección IV-A, donde se requiere de una inversión de tiempo y costos considerable, es entonces que se usan técnicas más simples como el análisis fotométrico para la determinación de metales pesados en muestras de agua, donde por lo general la prueba consiste en hacer reaccionar los metales en el medio con un agente fotométrico como la ditizona para posteriormente someter el complejo formado a un análisis con un fotómetro a una determinada longitud de onda para darnos una idea del contenido de un metal determinado en la muestra analizada [38].

\section{Técnicas electroquímicas}

Si bien con el tiempo se han desarrollado cada vez más técnicas en el ámbito del análisis de metales pesados a niveles mucho más bajos como los niveles de trazas y ultra-trazas como se vio en las secciones IV-A, IV-B, IV-C entre otras, una nueva técnica ha surgido que le permite al investigador medir estos metales y consiste en un método espectral de tipo electroquímico. Este método consiste en la combinación de un método electroquímico para un pre-enriquecimiento selectivo junto con un método espectroscópico para lograr el análisis tanto cualitativo como cuantitativo de estos contaminantes.

Hasta ahora métodos como la electrodeposición o electro adsorción han sido usados en conjunto con técnicas como la absorción atómica, espectrometría de Raman de superficie mejorada, fluorescencia de rayos X entre otras [39]-[42].

\section{MÉtodos De tratamiento}

Si bien los contaminantes que se generan y son desechados en los afluentes pueden ser clasificados como orgánicos e inorgánicos, no por ello se les puede tratar de manera similar. $\mathrm{Y}$ es que mientras para los contaminantes orgánicos se han desarrollado diferentes tratamientos tanto físicos, como biológicos o químicos, dichos métodos de tratamiento no son los más adecuados a la hora de tratar con contaminantes de tipo inorgánico como lo son los metales pesados, esto debido a que en el caso de estos últimos entran en juego otro tipo de cualidades que presentan como lo son la solubilidad, sus características de óxido-reducción y su capacidad en la formación de complejos, por lo cual la degradación de dichos metales plantea una mayor preocupación [23].

Es por eso que se han desarrollado técnicas especialmente para el tratamiento y remoción de este tipo de elementos contaminantes del medio acuoso, estas técnicas pueden considerarse como convencionales refiriéndonos a las técnicas normalmente usadas para la remoción de metales pesados (filtración por membrana, intercambio iónico, adsorción, 
precipitación química, electrocoagulación, coagulaciónfloculación entre otros) y las técnicas no convencionales las cuales hacen referencia a procesos innovadores para la remoción de metales pesados en aguas [23], [43].

\section{A. Filtración por membrana}

Esta técnica presenta altas eficiencias, ocupa poco espacio y es fácil de operar, entre sus mayores inconvenientes esta la generación de grandes cantidades de lodos que contienen metales. Se pueden clasificar las membranas de diversas maneras según sus características como su peso molecular de corte, el material de la membrana (si es sintético o polímeros naturales), permeabilidad, solubilidad del soluto y el solvente, superficie y espesor activo de la película, así como la carga de su superficie. Esta técnica generalmente es usada para recuperación de sales metálicas usadas en procesos de galvanoplastia, reciclaje de aceites, producción de alimentos y bebidas, entre otros [44]-[46]. Como se muestra en la Fig. 1, las técnicas como la microfiltración, nanofiltración y la ultrafiltración son ejemplos de algunas de las técnicas de remoción que son llevadas a cabo por el método de la filtración por membranas.

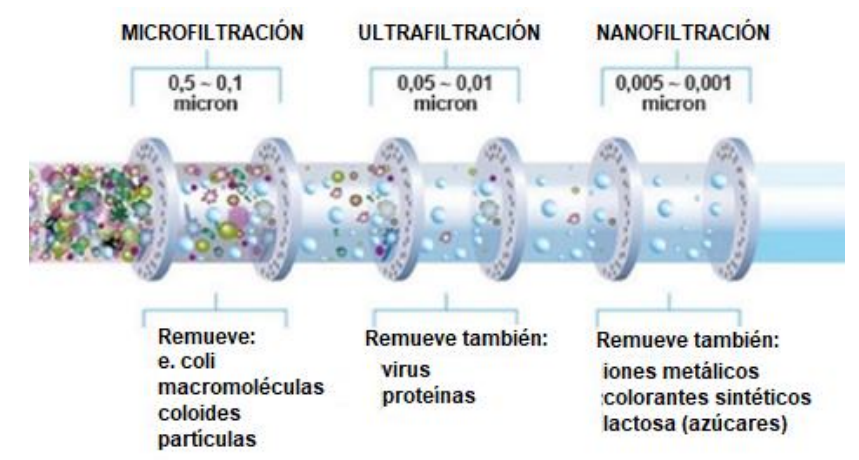

Fig. 1. Esquema de distintas técnicas de filtración por membrana [47].

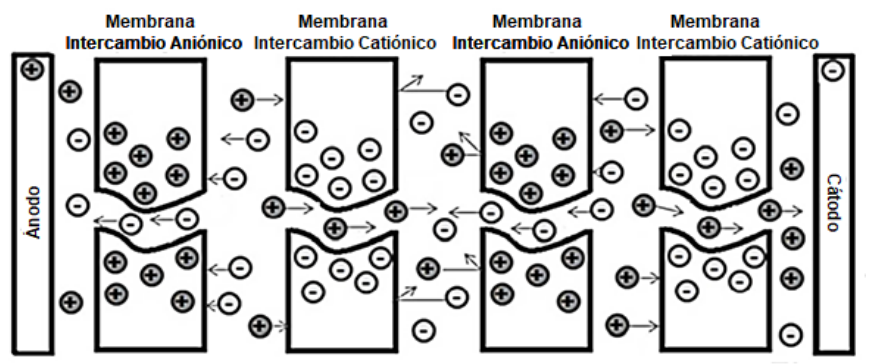

Fig. 2. Esquema de un sistema típico de electrodiálisis [51].

\section{1) Electrodiálisis}

La electrodiálisis es una técnica de descontaminación capaz de remover componentes iónicos de soluciones acuosas usando membranas permeables selectivas en un campo eléctrico constante tal que las membranas son colocadas ente dos electrodos tal como se muestra en la Fig. 2, esto hace que los cationes y aniones al pasar por las respectivas membranas tanto de intercambio catiónico como anicónico queden retenidos por estas [48]-[50].

\section{2) Osmosis inversa}

En el caso de la osmosis inversa usa membranas para la separación por difusión controlada o cibrado, se basa entonces en el principio de exclusión de tamaño y carga donde se usa una membrana semipermeable de un rango de tamaño de poro de entre 0.1 y $1.0 \mathrm{~nm}$ [45], [52].

\section{3) Nanofiltración}

La nanofiltración es una técnica de remoción relativamente reciente y requiere de presiones de funcionamiento en el rango de 10 - 50 bar. Por lo cual, las membranas usadas en este proceso son capaces de retener especies neutras con pesos moleculares menores a 200 o $300 \mathrm{~g} / \mathrm{mol}$. Es una técnica entre la osmosis inversa y la ultrafiltración y es un método fácil de manejar, de bajo coste energético, además requiere presiones más bajas que la osmosis inversa. Sin embargo su eficiencia está condicionada por el $\mathrm{pH}$, la presión, temperatura, la configuración y tendencia de la membrana [53]-[56].

\section{4) Ultrafiltración}

La ultrafiltración por otro lado es un proceso de fraccionamiento selectivo usando presiones de hasta 10 bar. Concentra sólidos en suspensión y solutos de peso molecular mayor a 1000 uma. Requiere de poca energía para el tratamiento de aguas residuales, además debido a las interacciones hidrofóbicas y electrostáticas de la membrana, la separación de las partículas de la solución acuosa es mucho más fácil. Para aumentar el proceso de ultrafiltración comúnmente se usan agentes químicos y poliméricos junto a la membrana y se le conoce a la técnica comúnmente como ultrafiltración mejorada con micelas [57], [58].

\section{B. Intercambio iónico}

Como su nombre lo indica, esta técnica consiste en un intercambio de iones de carga equivalente, en el cual una matriz sólida, generalmente una resina, se encarga de retener los iones metálicos en solución y en su lugar libera iones diferentes con igual carga como lo ilustra la Fig. 3. Es un proceso de separación física en el cual los iones intercambiados no sufren de alguna modificación química. Además si se lo compara con procesos de coagulación, esta técnica presenta mayores ventajas como que la producción de lodos por intercambio iónico es mucho más baja, tiende a ser más selectivo, además de una alta eficiencia en la remoción de iones metálicos en aguas contaminadas con los mismos [52], [59], [60].

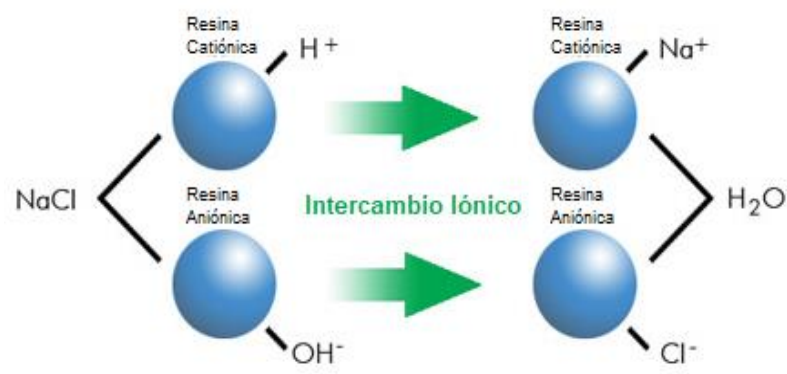

3. Esquema general del proceso de intercambio iónico [61].
Fig 


\section{Adsorción}

La adsorción es una técnica que presenta una remoción para gran variedad de contaminantes, así como una alta capacidad, cinética rápida y según el tipo de adsorbente a usar también presenta selectividad además de que por su estructura física también afectara su rendimiento. Es un método de conversión de masa en el cual los residuos son transferidos por interacciones químicas o físicas hacia los sitios activos presentes sobre el adsorbente usado. [62], [63]. Es así que la técnica de adsorción ilustrada en la Fig. 4, es un método de descontaminación fácil de usar, presenta bajo coste operacional, es flexible, de diseño simple y no produce contaminantes tóxicos, además de que la técnica permite la recuperación del adsorbente para ser usado con diferentes propósitos, convirtiendo esta técnica en un método aceptable a nivel ambiental [64].
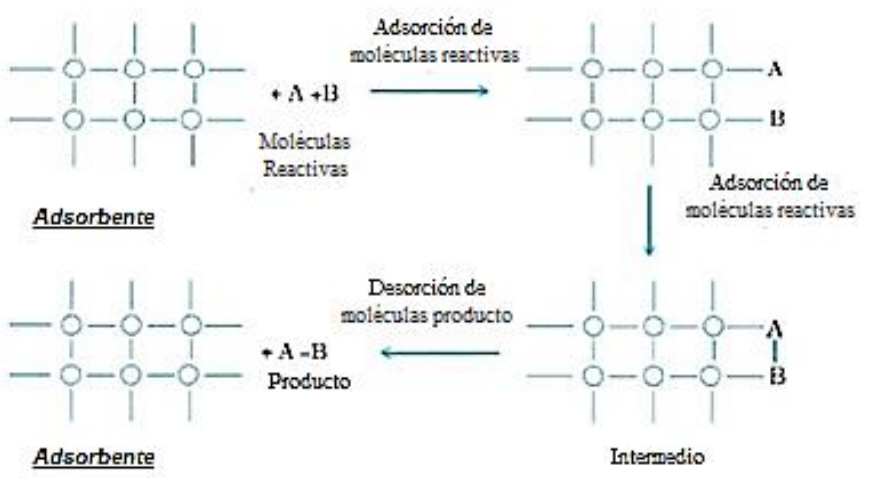

Fig. 4. Mecanismo de adsorción [65].

Entre las características que se debe tener presente a la hora de elegir un buen adsorbente está que tenga una gran área superficial, buena distribución del tamaño de poro, sus grupos funcionales, así como la polaridad del adsorbente, todo esto determinará la eficiencia del proceso de adsorción. Los carbones activados, arcillas, biopolímeros, zeolitas, perlas de sílice, plantas o desechos lignocelulósicos son algunos de los adsorbentes comúnmente usados para la remoción de colorantes iónicos, metales pesados, materiales radioactivos, entre otros contaminantes ya sea de tipo orgánico o inorgánico generados por los diferentes tipos de industrias, aunque en algunos casos este tipo de adsorbentes sufren procesos variados de modificación química [66]-[68].

\section{1) Carbón activado}

Entre los adsorbentes más usados, se encuentra el carbón activado el cual ha demostrado ser un adsorbente eficiente para la eliminación de una amplia variedad de contaminantes tanto orgánicos como inorgánicos de los ambientes acuosos. Esto debido a que su área superficial porosa puede ir dese los 500 hasta los $1500 \mathrm{~m}^{2} / \mathrm{g}$, así como la presencia de un amplio espectro de superficie funcional que la hace accesible a diferentes reactivos [69], [70]. Usualmente los adsorbentes de carbono se originan a partir de materiales carboneos como las biomasas, lignito y el propio carbón entre otros, donde el carbón es el más usado en la producción de carbón activado, sin embargo fuentes de biomasa más novedosas como el caso de la Glebionis Coronaria L. son usadas en la producción de carbón activado para la remoción de iones metálicos [71].

\section{2) Nanotubos de carbono}

Debido a su capacidad excepcional para la eliminación de diversos contaminantes orgánicos e inorgánicos y radionucleidos a partir de grandes volúmenes de aguas residuales, han despertado una amplia atención como un nuevo tipo de adsorbentes [72]. Los nanotubos de carbono están formados por hojas cilíndricas de grafito doblado en una estructura tipo tubo como se muestra en la Fig. 5. Se clasifican en dos tipos, (1) los nanotubos de carbono de pared simple (SWCNT) que consiste en una sola hoja de grafito y (2) los nanotubos de carbono de paredes múltiples (MWCNT) los cuales consisten en capas de múltiples hojas de grafito. El mecanismo por el cual interaccionan los nanotubos de carbono con los iones metálicos es debido a atracción electrostática, sorción-precipitación, intercambio iónico en interacción química. Son los mejores adsorbentes en el tratamiento de aguas residuales debido a sus propiedades mecánicas y magnéticas excelentes, su alta química y estabilidad térmica [54], [73].

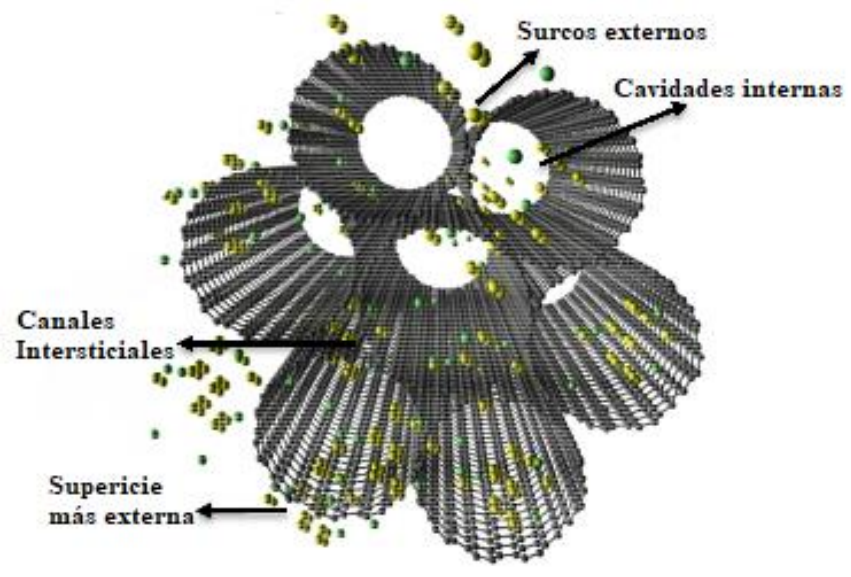

Fig. 5. Esquema de los nanotubos de carbono [74].

\section{Precipitación química}

A nivel industrial es la técnica más utilizada debido a su relativa facilidad para operarse, es una técnica económica y selectiva en cuanto a su proceso, sin embargo, a nivel de mantenimiento esta técnica si resulta costosa debido a la alta generación de lodos por parte de esta. Entre los tratamientos de precipitación química más usadas esta la precipitación por hidróxidos, la cual es una técnica de bajo costo, fácil control del pH y eliminación por floculación y sedimentación, aunque también se usa precipitación por sulfuros la cual presenta solubilidades bajas y precipitados no anfóteros. En algunos casos se han empleado agentes quelantantes aunque estos últimos presentan desventajas significativas, como la carencia de uniones necesarias, además de demasiados riesgos a nivel ambiental [75], [76]. Con el fin de formar un precipitado del metal, los agentes químicos adicionados a la solución, alteran el $\mathrm{pH}$ con el fin de impedir que el precipitado se disuelva en la solución, luego por proceso de sedimentación, estos precipitados son aislados y la solución restante es usada para otros propósitos [54], [77]. La precipitación química es más efectiva en la eliminación de iones metálicos como $\mathrm{Cu}$ (II), $\mathrm{Cd}$ (II), Mn (II) y Zn (II) [52]. 


\section{1) Precipitación por hidróxidos}

Es el método preferido en los procesos de remoción de metales pesados debido a su fácil manejo y bajo costo. La modificación del $\mathrm{pH}$ incrementa la formación de hidróxidos metálicos debido a su insolubilidad en medios alcalinos y en casos de capas de iones trivalentes se forman hidróxidos dobles para la remoción de metales pesados. Existen diferentes agentes precipitantes para este tipo de precipitación como el hidróxido de calcio o el hidróxido de sodio los cuales son muy útiles en el proceso de formación de precipitados metálicos [78], [79].

\section{2) Precipitación por sulfuros}

Es uno de los procesos más competentes entre los métodos de precipitación para la remoción de metales pesados de aguas residuales. La ventaja en el uso de la precipitación por sulfuros radica en que la solubilidad de los precipitados por sulfuro es más baja que la de los precipitados por hidróxido en un medio alcalino. Los precipitantes de sulfuro generalmente usados son sólidos como el sulfuro de hierro (FeS), acuosos como el sulfuro de sodio $\left(\mathrm{Na}_{2} \mathrm{~S}\right)$ o fuentes gaseosas de sulfuro como el sulfuro de hidrogeno $\left(\mathrm{H}_{2} \mathrm{~S}\right)$. El proceso de precipitación por sulfuro tiene buenas capacidades de asentamiento, remoción selectiva de metales y rápidos rangos de reacción. En algunos casos el uso de precipitación por sulfuros puede ocasionar la emisión de humos tóxicos en medio acido, por lo cual lo recomendable es usar esta técnica en condiciones básicas o neutrales [54], [80].

\section{E. Electrocoagulación}

Es un proceso que aplica los principios de la coagulaciónfloculación en un reactor electrolítico, el cual es un recipiente dotado de una fuente de corriente y varios electrodos que se encargan de aportar los iones desestabilizadores de partículas coloidales las cuales reemplazan las funciones de los compuestos químicos que se utilizan en el tratamiento convencional, por medio de inducción de una corriente eléctrica en el agua a través de placas metálicas paralelas de hierro o aluminio [54], [81].

\section{F. Coagulación-floculación}

Este es el método mediante el cual se logra la desestabilización de un coloide para su posterior aglomeración. En una primera etapa, la coagulación elimina la doble capa eléctrica que caracteriza a los coloides y con la floculación se aglomeran los coloides mediante la atracción de partículas con el aglutinamiento de los floculantes. Entre los factores más importantes a tener en cuenta para esta técnica está la dosis química apropiada, el pH, temperatura, alcalinidad, el efecto energético de la mezcla y el tiempo de la misma. Los coagulantes químicos comúnmente usados en el tratamiento de aguas residuales incluyen alumbre $\left(\mathrm{Al}_{3}\left(\mathrm{SO}_{4}\right)_{3} \cdot 18 \mathrm{H}_{2} \mathrm{O}\right)$, cloruro férrico hexahidratado $\left(\mathrm{FeCl}_{3} \cdot 6 \mathrm{H}_{2} \mathrm{O}\right)$, sulfato férrico $\left(\mathrm{Fe}_{2}\left(\mathrm{SO}_{4}\right)_{3}\right)$, sulfato ferroso heptahidratado $\left(\mathrm{FeSO}_{4} \cdot 7 \mathrm{H}_{2} \mathrm{O}\right)$ y cal $\left(\mathrm{Ca}(\mathrm{OH})_{2}\right)[82]-[85]$.

\section{G. Electrofloculación}

Es un proceso químico basado en la adición electrolítica de iones metálicos y su eficiencia en la remoción de los contaminantes se debe a que son arrastrados por las burbujas de gas $\left(\mathrm{H}_{2}\right.$ y $\left.\mathrm{O}_{2}\right)$ que se producen en el sistema y por lo cual salen a flote en la superficie [43]. Juega un rol muy importante en el tratamiento de aguas, además de combinar la oxidación electrolítica parcial y la precipitación electrotérmica o electroquímica, es fácil de usar y puede emplearse con diferentes tipos de contaminantes sin la formación de lodos residuales por uso de reactivos químicos. La electrofloculación ocurre en tres fases: (1) la generación de pequeñas burbujas de gas después del contacto con partículas electrolíticas suspendidas; (2) la absorción de las burbujas de gas por la superficie de las partículas electrolíticas; y (3) un incremento del agregado partícula-burbuja [86].

\section{H. Flotación}

El proceso de flotación de iones se basa en impartir las especies iónicas del metal en aguas residuales hidrófobas mediante el uso de agentes activos de superficie (tensoactivos) y la posterior eliminación de estas especies hidrófobas por medio de burbujas de aire [60], [87]-[89].

\section{Fotocatálisis en la degradación de metales pesados}

Esta técnica se basa en la transferencia de carga a través de la interfaz entre el semiconductor y la solución acuosa contaminada, donde la conductividad aumenta con la temperatura y se genera un par electrón-hueco, ocurriendo la adsorción de los fotones y la distribución de diferentes estados electrónicos en la superficie, degradando moléculas orgánicas colorantes y metales pesados [90].

\section{CONCLUSIONES}

Conforme pasa el tiempo nuevas y diversas formas de tratamiento para aguas contaminadas van surgiendo, lo cual permite una mayor gama de posibilidades en el ámbito de los procesos de descontaminación de los recursos hídricos. Algunos de estos procesos relativamente no demandan grandes procesos de limpieza para lograr su cometido y es necesario que el efecto de los metales pesados sobre los organismos sea detenido por medio de las técnicas estudiadas en pro de mejorar la salud tanto de seres humanos como de los ecosistemas.

\section{AGRADECIMIENTOS}

Los Autores agradecen a la Unidad de Análisis Industriales y al Departamento de Química de la Universidad del Cauca por su colaboración en el desarrollo de esta investigación.

\section{REFERENCIAS}

[1] L. F. Londoño Franco, P. T. Londoño Muñoz, and F. G. Muñoz Garcia, "Los riesgos de los metales pesados en la salud humana y animal," Biotecnoloía en el Sect. Agropecu. y Agroindustrial, vol. 14, no. 2, p. $145,2016$.

[2] J. Contreras Pérez, C. L. Mendoza, and A. Gómez, "Determinación de metales pesados en aguas y sedimentos del río Haina," Cienc. Soc., vol. 
29, no. 1, pp. 38-71, 2004.

[3] O. Cartaya, I. Reynaldo, and C. Peniche, "Cinética De Adsorción De Iones Cobre (II) Por Una Mezcla De Oligogalacturónidos," Rev. Iberoam. Polímero Vol. Iberoam. Polim, vol. 9, no. 95, pp. 473-479, 2008.

[4] R. Vilchez, "Eliminación de metales pesados de aguas subterráneas mediante sistemas de lechos sumergidos: Estudio microbiológico de las biopeliculas," Universidad de Granada, 2005.

[5] Y. C. Reyes, I. Vergara, O. E. Torres, M. Díaz, and E. E. González, "Contaminación Por Metales Pesados: Implicaciones En Salud, Ambiente Y Seguridad Alimentaria," Ing. Investig. y Desarro., vol. 16, no. 2, pp. 66-77, 2016.

[6] C. Azpilicueta, L. Pena, and S. Gallego, "Los metales y las plantas: entre la nutrición y la toxicidad," Cienc. Hoy, vol. 20, p. 5, 2010.

[7] S. Suthersan, "Evaluación de las tecnologías de tratamiento de aguas subterráneas contaminadas con Cromo," p. 105, 2007.

[8] C. Nava-Ruíz and M. Méndez-Armenta, "Efectos neurotóxicos de metales pesados (cadmio, plomo, arsénico y talio)," Arch. Neurociencias, vol. 16, no. 3, pp. 140-147, 2011.

[9] C. Tejada-Tovar, Á. Villabona-Ortiz, and L. Garcés-Jaraba, "Adsorción de metales pesados en aguas residuales usando materiales de origen biológico," Tecno Lógicas, vol. 18, no. 34, pp. 109-123, 2015.

[10] S. E. Manahan, Introducción a la química ambiental. Editorial Reverté, 2007.

[11] V. E. Gomez Rengifo, J. A. Velásquez Jiménez, and G. C. Quintana Marín, "Lignina como adsorbente de metales pesados," Rev. Investig. Apl., vol. 7, no. 2, pp. 74-85, 2013.

[12] P. Trivedi and L. Axe, "Modeling Cd and Zn sorption to hydrous metal oxides," Environ. Sci. Technol., vol. 34, no. 11, pp. 2215-2223, 2000.

[13] M. A. Ahmed, S. M. Ali, S. I. El-Dek, and A. Galal, "Magnetitehematite nanoparticles prepared by green methods for heavy metal ions removal from water," Mater. Sci. Eng. B Solid-State Mater. Adv. Technol., vol. 178, no. 10, pp. 744-751, 2013.

[14] D. Mohan, K. P. Singh, and V. K. Singh, "Trivalent chromium removal from wastewater using low cost activated carbon derived from agricultural waste material and activated carbon fabric cloth," $J$. Hazard. Mater., vol. 135, no. 1-3, pp. 280-295, 2006.

[15] O. C. S. Al Hamouz, M. Estatie, and T. A. Saleh, "Removal of cadmium ions from wastewater by dithiocarbamate functionalized pyrrole based terpolymers," Sep. Purif. Technol., vol. 177, pp. 101-109, 2017.

[16] C. Y. Wu, H. Mouri, S. S. Chen, D. Z. Zhang, M. Koga, and J. Kobayashi, "Removal of trace-amount mercury from wastewater by forward osmosis," J. Water Process Eng., vol. 14, pp. 108-116, 2016.

[17] A. M. Mohammad, T. A. Salah Eldin, M. A. Hassan, and B. E. ElAnadouli, "Efficient treatment of lead-containing wastewater by hydroxyapatite/chitosan nanostructures," Arab. J. Chem., vol. 10, no. 5, pp. 683-690, 2017.

[18] Ministerio de la Presidencia, "Real Decreto 140/2003, de 7 de febrero, por el que se establecen los criterios sanitarios de la calidad del agua de consumo humano.," Bol. Of. del estado, pp. 7228-7245, 2003.

[19] Instituto colombiano de normas técnicas y certificación, "Norma Técnica Colombiana Ntc 813,” vol. 813, pp. 1-10, 2011.

[20] O. US EPA, "National Primary Drinking Water Regulations." [Online]. Available: https://www.epa.gov/ground-water-and-drinkingwater/national-primary-drinking-water-regulations\#one. [Accessed: 09Apr-2019].

[21] C. García, J. L. Moreno, M. Teresa, and A. Polo, "Metales pesados y sus implicaciones en la calidad del suelo," Cienc. y Medio Ambient., p. 14, 2002.

[22] B. Wang, Y. Zhu, Z. Bai, R. Luque, and J. Xuan, "Functionalized chitosan biosorbents with ultra-high performance, mechanical strength and tunable selectivity for heavy metals in wastewater treatment," Chem. Eng. J., vol. 325, pp. 350-359, 2017

[23] C. F. Carolin, P. S. Kumar, A. Saravanan, G. J. Joshiba, and M Naushad, "Efficient techniques for the removal of toxic heavy metals from aquatic environment: A review," J. Environ. Chem. Eng., vol. 5, no. 3, pp. 2782-2799, 2017.

[24] C. M. Mejia Dominguez, "Metales Pesados En Suelos Y Plantas: Contaminación Y Fitotoxicidad," 2011.

[25] O. L. B. Moreno, "Estudio de diferentes metodologías para determinar la biodisponibilidad de cadmio y arsénico en suelos y su relación con la concentración en plantas," Nova, vol. 6, no. 9, pp. 35-39, 2008.

[26] L. E. Cortés, F. J. Martin, and M. M. Sarria, "Evaluación de la toxicidad de metales pesados en dos suelos agrícolas de Colombia mediante bioensayos Evaluation of heavy metal toxicity in two Colombian agricultural soils using," 2017.

[27] "What is a LD50 and LC50?: OSH Answers." [Online]. Available: http://www.ccohs.ca/oshanswers/chemicals/ld50.html. [Accessed: 20May-2018].

[28] C. Baird, Quimica ambiental. University of Western. Ontario: Editorial Reverté, 2001.

[29] X. Domènech and J. Peral, Química ambiental de sistemas terrestres. Barcelona, España: Reverté, 2006.

[30] D. W. O'Connell, C. Birkinshaw, and T. F. O'Dwyer, "Heavy metal adsorbents prepared from the modification of cellulose: A review," Bioresour. Technol., vol. 99, no. 15, pp. 6709-6724, 2008.

[31] K. Leopold, A. Philippe, K. Wörle, and G. E. Schaumann, "Analytical strategies to the determination of metal-containing nanoparticles in environmental waters," TrAC Trends Anal. Chem., vol. 84, pp. 107120, Nov. 2016.

[32] P. Pohl, P. Jamroz, M. Welna, A. Szymczycha-Madeja, and K. Greda, "Chemical-vapor generation of transition metals through the reaction with tetrahydroborate in recent achievements in analytical atomic spectrometry," TrAC Trends Anal. Chem., vol. 59, pp. 144-155, Jul. 2014.

[33] A. T. Reis, C. M. Davidson, C. Vale, and E. Pereira, "Overview and challenges of mercury fractionation and speciation in soils," $\operatorname{TrAC}$ Trends Anal. Chem., vol. 82, pp. 109-117, Sep. 2016.

[34] S. L. C. Ferreira et al., "Atomic absorption spectrometry - A multi element technique," TrAC Trends Anal. Chem., vol. 100, pp. 1-6, Mar 2018.

[35] M. Shen, L. Chen, W. Han, and A. Ma, "Methods for the determination of heavy metals in indocalamus leaves after different preservation treatment using inductively-coupled plasma mass spectrometry," Microchem. J., vol. 139, pp. 295-300, Jun. 2018

[36] A. Harris, S. J. Xanthos, J. K. Galiotos, and C. Douvris, "Investigation of the metal content of sediments around the historically polluted Potomac River basin in Washington D.C., United States by inductively coupled plasma-optical emission spectroscopy (ICP-OES)," Microchem. J., vol. 142, pp. 140-143, Nov. 2018

[37] M. I. S. Rojas, "La espectrometría de emisión con fuente de plasma de acoplamiento inductivo Inductively coupled plasma emission spectrometry," Mater. Construcción, vol. 36, no. 202, pp. 31-46, 1986.

[38] B. Romberg and H. Müller, "Photometric screening-test for heavy metals under flow injection conditions using extractive determination with dithizone," Anal. Chim. Acta, vol. 353, no. 2-3, pp. 165-172, Oct. 1997.

[39] S.-H. Chen et al., "Electrochemical spectral methods for trace detection of heavy metals: A review," TrAC Trends Anal. Chem., vol. 106, pp. 139-150, Sep. 2018.

[40] E. Bulska, "Analytical advantages of using electrochemistry for atomic spectrometry," Pure Appl. Chem., vol. 73, no. 1, pp. 1-7, Jan. 2001.

[41] S. Wang, E. S. Forzani, and N. Tao, "Detection of Heavy Metal Ions in Water by High-Resolution Surface Plasmon Resonance Spectroscopy Combined with Anodic Stripping Voltammetry," Anal. Chem., vol. 79, no. 12, pp. 4427-4432, Jun. 2007.

[42] E. Marguí, R. Van Grieken, C. Fontàs, M. Hidalgo, and I. Queralt, "Preconcentration Methods for the Analysis of Liquid Samples by XRay Fluorescence Techniques," Appl. Spectrosc. Rev., vol. 45, no. 3, pp. 179-205, May 2010.

[43] D. I. C. Rubio, R. A. M. Calderón, A. P. Gualtero, D. R. Acosta, and I. J. S. Rojas, "Tratamientos para la Remoción de Metales Pesados Comúnmente Presentes en Aguas Residuales Industriales. Una Revisión," Rev. Ing. y Región, vol. 13, no. 1, pp. 73-90, 2015.

[44] J. Taylor and M. Wiesner, Calidad y tratamiento del agua : manual de suministros de agua comunitaria. Madrid, España: McGraw-Hill, 2002.

[45] N. L. Nemerow and A. Dasgupta, Tratamiento de vertidos industriales y peligrosos. Ediciones Díaz de Santos, 2000.

[46] Y. Ji, "Membrane technologies for water treatment and reuse in the gas and petrochemical industries," in Advances in Membrane Technologies for Water Treatment, Elsevier, 2015, pp. 519-536. 
[47] "Nanofiltration Device Pictures to Pin on Pinterest - PinsDaddy." [Online]. Available: http://www.pinsdaddy.com/nanofiltrationdevice_JzLzpW2NQPbU5Wb4*hNS1hbCHndH1lhn50tVQFop6gc/. [Accessed: 10-Apr-2019].

[48] E. Brillas and P.-L. Cabot, Trends in electrochemistry and corrosion at the beginning of the 21st century: dedicated to professor Dr. Josep M. Costa on the occasion of his 70th birthday. Publicacions Universitat de Barcelona, 2004.

[49] Y. Liu, J. Yan, D. Yuan, Q. Li, and X. Wu, "The study of lead removal from aqueous solution using an electrochemical method with a stainless steel net electrode coated with single wall carbon nanotubes," Chem. Eng. J., vol. 218, pp. 81-88, Feb. 2013.

[50] C.-V. Gherasim, J. Křivčík, and P. Mikulášek, "Investigation of batch electrodialysis process for removal of lead ions from aqueous solutions," Chem. Eng. J., vol. 256, pp. 324-334, Nov. 2014.

[51] T. Scarazzato, Z. Panossian, J. A. S. Tenório, V. Pérez-Herranz, and D. C. R. Espinosa, "A review of cleaner production in electroplating industries using electrodialysis," J. Clean. Prod., vol. 168, pp. 1590$1602,2017$.

[52] M. Bilal et al., "Waste biomass adsorbents for copper removal from industrial wastewater-A review," J. Hazard. Mater., vol. 263, pp. 322-333, Dec. 2013.

[53] M. P. González et al., "Effect of phosphoric and hydrofluoric acid on the charge density of a nanofiltration membrane," Desalination, vol. 200, no. 1-3, pp. 361-363, Nov. 2006.

[54] F. Fu and Q. Wang, "Removal of heavy metal ions from wastewaters: A review," J. Environ. Manage., vol. 92, no. 3, pp. 407-418, Mar. 2011.

[55] W. Tao et al., "Influence of silver nanoparticles on heavy metals of pore water in contaminated river sediments," Chemosphere, vol. 162, pp. 117-124, Nov. 2016.

[56] S. S. Hosseini, E. Bringas, N. R. Tan, I. Ortiz, M. Ghahramani, and M. A. Alaei Shahmirzadi, "Recent progress in development of high performance polymeric membranes and materials for metal plating wastewater treatment: A review," J. Water Process Eng., vol. 9, pp. 78110, Feb. 2016.

[57] A. A. Mungray, S. V. Kulkarni, and A. K. Mungray, "Removal of heavy metals from wastewater using micellar enhanced ultrafiltration technique: a review," Cent. Eur. J. Chem., vol. 10, no. 1, pp. 27-46, Feb. 2012.

[58] J. Landaburu-Aguirre, V. García, E. Pongrácz, and R. L. Keiski, "The removal of zinc from synthetic wastewaters by micellar-enhanced ultrafiltration: statistical design of experiments," Desalination, vol. 240, no. 1-3, pp. 262-269, May 2009.

[59] T. M. Zewail and N. S. Yousef, "Kinetic study of heavy metal ions removal by ion exchange in batch conical air spouted bed," Alexandria Eng. J., vol. 54, no. 1, pp. 83-90, Mar. 2015.

[60] Z. Hubicki and D. Koodynsk, "Selective Removal of Heavy Metal Ions from Waters and Waste Waters Using Ion Exchange Methods," in Ion Exchange Technologies, InTech, 2012.

[61] "Water quality information - Water Purification Technologies - Ion exchange | APEC Water." [Online]. Available: https://www.freedrinkingwater.com/water-education/quality-waterfiltration-method-ion-exchange.htm. [Accessed: 12-Apr-2019].

[62] Y. Chang, J.-Y. Lai, and D.-J. Lee, "Thermodynamic parameters for adsorption equilibrium of heavy metals and dyes from wastewaters: Research updated," Bioresour. Technol., vol. 222, pp. 513-516, Dec. 2016.

[63] A. T. Ojedokun and O. S. Bello, "Sequestering heavy metals from wastewater using cow dung," Water Resour. Ind., vol. 13, pp. 7-13, Mar. 2016.

[64] A. Demirbas, "Heavy metal adsorption onto agro-based waste materials: A review," J. Hazard. Mater., vol. 157, no. 2-3, pp. 220-229, Sep. 2008.

[65] B. E. Saad, "Chapter 3 PRINCIPLES OF ADSORPTION," pp. 41-65, 2016.

[66] A. Ewecharoen, P. Thiravetyan, E. Wendel, and H. Bertagnolli, "Nickel adsorption by sodium polyacrylate-grafted activated carbon," J. Hazard. Mater., vol. 171, no. 1-3, pp. 335-339, Nov. 2009.

[67] W. S. Wan Ngah, L. C. Teong, and M. A. K. M. Hanafiah, "Adsorption of dyes and heavy metal ions by chitosan composites: A review," Carbohydr. Polym., vol. 83, no. 4, pp. 1446-1456, Feb. 2011.

[68] P. O. Boamah et al., "Sorption of heavy metal ions onto carboxylate chitosan derivatives-A mini-review," Ecotoxicol. Environ. Saf., vol. 116, pp. 113-120, Jun. 2015.

[69] G. Mezohegyi, F. P. van der Zee, J. Font, A. Fortuny, and A. Fabregat, "Towards advanced aqueous dye removal processes: A short review on the versatile role of activated carbon," J. Environ. Manage., vol. 102, pp. 148-164, Jul. 2012.

[70] M. Karnib, A. Kabbani, H. Holail, and Z. Olama, "Heavy Metals Removal Using Activated Carbon, Silica and Silica Activated Carbon Composite," Energy Procedia, vol. 50, pp. 113-120, 2014.

[71] H. Tounsadi et al., "Highly efficient activated carbon from Glebionis coronaria L. biomass: Optimization of preparation conditions and heavy metals removal using experimental design approach," J. Environ. Chem. Eng., vol. 4, no. 4, pp. 4549-4564, Dec. 2016.

[72] X. Ren, C. Chen, M. Nagatsu, and X. Wang, "Carbon nanotubes as adsorbents in environmental pollution management: A review," Chemical Engineering Journal, vol. 170, no. 2-3. pp. 395-410, Jun2011.

[73] Ihsanullah et al., "Effect of acid modification on adsorption of hexavalent chromium $(\mathrm{Cr}(\mathrm{VI}))$ from aqueous solution by activated carbon and carbon nanotubes," Desalin. Water Treat., vol. 57, no. 16, pp. 7232-7244, Apr. 2016.

[74] G. Ersan, O. G. Apul, F. Perreault, and T. Karanfil, "Adsorption of organic contaminants by graphene nanosheets: A review," Water Res. vol. 126, pp. 385-398, Dec. 2017.

[75] J. L. Huisman, G. Schouten, and C. Schultz, "Biologically produced sulphide for purification of process streams, effluent treatment and recovery of metals in the metal and mining industry," Hydrometallurgy, vol. 83, no. 1-4, pp. 106-113, Sep. 2006

[76] Q. Chen, Z. Luo, C. Hills, G. Xue, and M. Tyrer, "Precipitation of heavy metals from wastewater using simulated flue gas: Sequent additions of fly ash, lime and carbon dioxide," Water Res., vol. 43, no. 10, pp. 2605-2614, Jun. 2009.

[77] M. A. Hashim, S. Mukhopadhyay, J. N. Sahu, and B. Sengupta, "Remediation technologies for heavy metal contaminated groundwater," J. Environ. Manage., vol. 92, no. 10, pp. 2355-2388, Oct. 2011.

[78] J. Z. Zhou et al., "Effective Self-Purification of Polynary Metal Electroplating Wastewaters through Formation of Layered Double Hydroxides," Environ. Sci. Technol., vol. 44, no. 23, pp. 8884-8890, Dec. 2010.

[79] S. V. Jadhav, C. R. Gadipelly, K. V. Marathe, and V. K. Rathod, "Treatment of fluoride concentrates from membrane unit using salt solutions," J. Water Process Eng., vol. 2, pp. 31-36, Jun. 2014.

[80] A. E. Lewis, "Review of metal sulphide precipitation," Hydrometallurgy, vol. 104, no. 2, pp. 222-234, Sep. 2010.

[81] G. Morante, "Electrocoagulación de aguas residuales," Rev. Colomb. Física, vol. 34, no. 2, pp. 484-487, 2002.

[82] M. Visa, "Synthesis and characterization of new zeolite materials obtained from fly ash for heavy metals removal in advanced wastewater treatment," Powder Technol., vol. 294, pp. 338-347, Jun. 2016.

[83] D. Ghernaout et al., "Brownian Motion and Coagulation Process," Am. J. Environ. Prot. Spec. Issue Clean. Sustain. Prod., vol. 4, no. 1, pp. 1$15,2015$.

[84] I. M. Ismail, A. S. Fawzy, N. M. Abdel-Monem, M. H. Mahmoud, and M. A. El-Halwany, "Combined coagulation flocculation pre treatment unit for municipal wastewater," J. Adv. Res., vol. 3, no. 4, pp. 331-336, Oct. 2012.

[85] F. Renault, B. Sancey, P.-M. Badot, and G. Crini, "Chitosan for coagulation/flocculation processes - An eco-friendly approach," Eur. Polym. J., vol. 45, no. 5, pp. 1337-1348, May 2009.

[86] M. S. Melchiors, M. Piovesan, V. R. Becegato, V. A. Becegato, E. B. Tambourgi, and A. T. Paulino, "Treatment of wastewater from the dairy industry using electroflocculation and solid whey recovery," J. Environ. Manage., vol. 182, pp. 574-580, Nov. 2016.

[87] H. Polat and D. Erdogan, "Heavy metal removal from waste waters by ion flotation," J. Hazard. Mater., vol. 148, no. 1-2, pp. 267-273, Sep. 2007.

[88] F. S. Hoseinian, M. Irannajad, and A. J. Nooshabadi, "Ion flotation for removal of $\mathrm{Ni}(\mathrm{II})$ and $\mathrm{Zn}(\mathrm{II})$ ions from wastewaters," Int. J. Miner. Process., vol. 143, pp. 131-137, Oct. 2015.

[89] M. H. Salmani, M. Davoodi, M. H. Ehrampoush, M. T. Ghaneian, and M. H. Fallahzadah, "Removal of cadmium (II) from simulated wastewater by ion flotation technique," Iranian J. Environ. Health Sci. 
Eng., vol. 10, no. 1, p. 16, 2013.

[90] C. Páez and G. Taborda, "La fotocatálisis: aspectos fundamentales para una buena remoción de contaminantes," Rev. la Univ. Caldas, vol. 26, no. $1-2$, pp. 71-88, 2006.

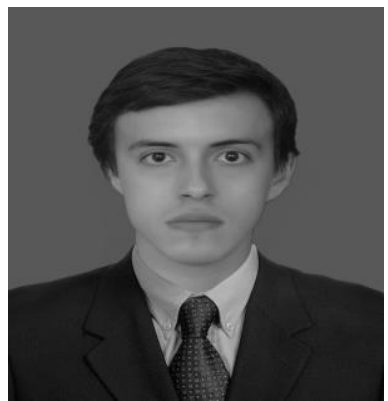

Santiago Eduardo Pabón Guerrero. Investigador del Grupo de Investigación en Química Analítica Ambiental de la Universidad del Cauca. Candidato a Químico de la Universidad del Cauca, Popayán, Colombia. Sus áreas de interés son: Química ambiental, nanomateriales y recursos renovables.

ORCID: https://orcid.org/0000-00020157-0166.

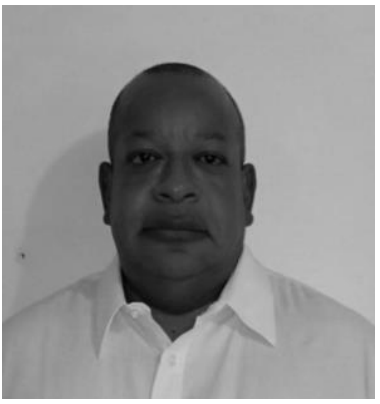

Ricardo Benítez Benítez. Investigador del Grupo de Investigación en Química de Productos Naturales de la Universidad del Cauca, Profesor Titular del departamento de Química de la Universidad del Cauca, Popayán, Colombia. Licenciado en Bioquímica por la Universidad del Valle, Cali, Colombia. Maestría en Ciencias Químicas de la Universidad del Valle, Cali, Colombia y Doctor en Ciencia y Tecnología de Alimentos de la Universidad de Lleida, Lleida, España y cuenta con más de 30 años de experiencia industrial, docente y de investigación. Sus áreas de interés son: Bioquímica, actividad enzimática y recursos renovables.

ORCID: https://orcid.org/0000-0002-8999-3680.

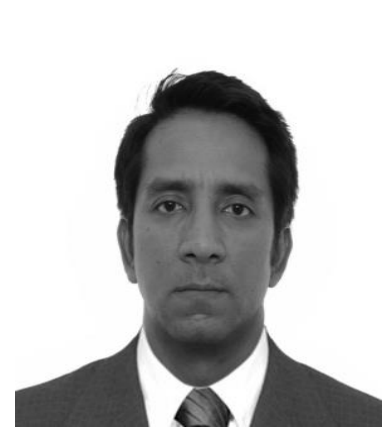

Rodrigo Andrés Sarria Villa. Investigador del Grupo de Investigación en Química Analítica Ambiental de la Universidad del Cauca y Profesor Ocasional tiempo completo del Departamento de Química de la Universidad del Cauca, Popayán, Colombia. Químico de la Universidad del Cauca, Popayán, Colombia. Maestría en Ciencias Químicas de la Universidad del Valle, Cali, Colombia y Doctor en Ciencias Químicas de la Universidad del Valle, Cali, Colombia, cuenta con 3 años de experiencia como docente, 10 años de experiencia como investigador y es miembro de la sociedad ambiental SETAC. Sus áreas de interés son: Química ambiental, toxicología y recursos renovables.

ORCID: https://orcid.org/0000-0003-1295-7865.

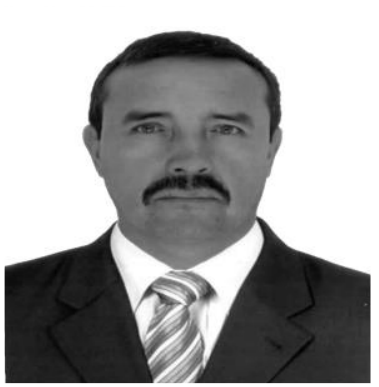

José Antonio Gallo Corredor. Investigador y Coordinador del Grupo de Investigación en Química Analítica Ambiental de la Universidad del Cauca, Popayán, Colombia. Licenciado en Ciencias de la Educación Química y Biología de la Universidad Pedagógica y Tecnológica de Colombia, Tunja, Colombia. Maestría en Ciencias Químicas de la Universidad del Valle, Cali, Colombia y Candidato a doctor en Ciencias de la Universidad del Cauca, Popayán, Colombia y cuenta con 20 años de experiencia como docente e investigador. Sus áreas de interés son: Contaminación por actividad minera y aplicaciones en residuos agroforestales. ORCID: https://orcid.org/0000-0002-4861-2837. 